\title{
Chest computed tomography findings of COVID-19 in children younger than 1 year: a systematic review
}

\author{
Alireza Ghodsi ${ }^{1}$. Moniba Bijari ${ }^{1}$ - Seyed Ali Alamdaran ${ }^{2}$. Amin Saberi ${ }^{3}$ - Elnaz Mahmoudabadi ${ }^{1}$. \\ Mohammad Reza Balali ${ }^{4}$. Sara Ghahremani ${ }^{5}$ (I)
}

Received: 15 November 2020 / Accepted: 25 February 2021 / Published online: 7 May 2021

(c) Children's Hospital, Zhejiang University School of Medicine 2021

\begin{abstract}
Background The aim of this systematic review is to evaluate the chest computed tomography (CT) findings in infants with confirmed COVID-19 infection by providing a comprehensive review of the existing literature.

Data sources A systematic search was conducted on PubMed and Embase from the onset of the COVID-19 outbreak to October 20, 2020, for studies that discussed the chest CT findings in infants younger than 1 year with COVID-19 infection. Results A total of 35 studies comprising 70 COVID-19 (58.5\% boys) confirmed infants were included. The mean age of the included patients was 4.1 months with a range of 1 day to 12 months. Chest CT scans showed bilateral abnormalities in 34 patients, and unilateral lung involvement in 25 patients. Ground-glass opacities (GGO) (71.43\%) were found to be the most prevalent chest CT manifestation, followed by peribronchial thickening (60\%), linear or band-shaped opacities (32.8\%), consolidation (28.57\%), nodule (18.57\%), effusion (7.14\%) and focal lucency $(7.14 \%)$.

Conclusions GGO and peribronchial thickening were the most prevalent findings in the infants' chest CT scans. Linear or band-shaped opacities, consolidation, and pulmonary nodules are more common in infants than in adults. These findings suggest that the disease is more likely to be presented as an atypical pneumonia (peribronchial thickening and linear or bandshaped opacities) in this age group. Other chest CT scan manifestations can be classified as typical COVID-19 infection (peripheral GGO), lobar pneumonia (consolidation) and opportunistic infections (pulmonary nodules).
\end{abstract}

Keywords Computerized tomography $\cdot$ COVID-19 $\cdot$ Infant $\cdot$ SARS-CoV-2 $\cdot$ Systematic review

\section{Introduction}

SARS-CoV-2 primarily affects the respiratory system, and its main clinical manifestations are fever, cough, myalgia, and shortness of breath [1]. Early diagnosis of the disease is of paramount importance, not only in symptomatic patients

Sara Ghahremani

ghahremanis@mums.ac.ir

1 Student Research Committee, Faculty of Medicine, Mashhad University of Medical Sciences, Mashhad, Iran

2 Radiology Department, Faculty of Medicine, Mashhad University of Medical Sciences, Mashhad, Iran

3 Faculty of Medicine, Mashhad University of Medical Sciences, Azadi Sq, Mashhad, Iran

4 Pediatrician, Hasheminezhad Hospital, Mashhad, Iran

5 Department of Pediatrics, Faculty of Medicine, Mashhad University of Medical Sciences, Mashhad, Iran but also in asymptomatic ones that may be in their incubation period and act as a source of transmission. The gold standards for the diagnosis of COVID-19 infection are nucleic acid testing (NAT) or real-time reverse transcriptasepolymerase chain reaction (RT-PCR) [2]. However, these two tests have relatively low sensitivity, i.e., high false-negative results [3]. Moreover, the number of available kits for these tests is limited [4]. Radiological examination plays a key role in the diagnosis of COVID-19 as well as in the diagnosis of other respiratory diseases. Among different imaging modalities, chest computed tomography (CT) has been the major imaging technique for the diagnosis and follow-up of patients with COVID-19 [5]. There is some evidence that clinical symptoms along with $\mathrm{CT}$ findings are suggestive for COVID-19, even in the presence of a negative PCR test [6]. The imaging findings of chest CT scan can be diverse and variable from one patient to another. Therefore, it is necessary to fully understand the COVID-19-associated CT scan findings for a more accurate decision-making process. 
A systematic review comparing chest $\mathrm{CT}$ findings in children and adult populations concluded that children are more likely to have normal reports or atypical lung involvement [7]. However, to the best of our knowledge, there is no study in which chest CT findings are compared among different age groups of the pediatric population. Infants below 1 year are considered a vulnerable group in this population, for whom there are very few studies in this regard, while several case reports, case series, and cross-sectional studies have reported CT scan findings of COVID-19 in children. Therefore, the present study aimed to systematically review the existing literature on specific CT scan findings of COVID-19 in young infants.

\section{Methods}

\section{Study protocol}

The present study's protocol follows the recommendations established by the Preferred Reporting Items for Systematic reviews and Meta-Analysis (PRISMA) statement [8].

\section{Search strategy}

Based on our research question of "What are the specific features of novel coronavirus infection in chest CT of infants?", a systematic literature search was done using two major electronic databases: PubMed and Embase. We searched for studies published from December 1st, 2019 to October 20, 2020, using the following search terms: ["COVID-19" OR “coronavirus" OR "SARS-CoV-2" OR "2019-nCoV"] AND ["neonate" OR "infant" OR "newborn"] AND ["computed tomography" OR "CT-scan" OR "CT"]. No language restrictions were set. Additional articles were added by a manual search of the reference lists of the selected articles and comprehensive review articles.

\section{Selection of studies}

After removing the duplicates, two investigators (MB and AG) independently screened the titles and abstracts of the remaining studies for the inclusion criteria. We included all studies that investigated CT scan findings of confirmed COVID-19 infection [with reverse transcriptase-polymerase chain reaction (RT-PCR) testing] in infants under 1 year old. We included case reports and case series that reported chest CT imaging findings of infants with COVID-19 to widen our search. Considering the exclusion criteria, we excluded studies reported as editorials, abstracts or conference proceedings, opinion articles, multimedia files, or studies on non-human species. Moreover, studies on other coronavirusrelated diseases were excluded. We also excluded the articles which had reported on a mixed adult-child population in which imaging results could not be extracted for the infants' group.

\section{Data extraction and synthesis}

Data extraction was performed by two investigators (AG and MB) using a standardized data extraction form. A pediatric radiologist (AA) screened all available chest $\mathrm{CT}$ scans in the included articles and reported independently. The disagreements and inconsistencies were resolved by consensus. Then, we extracted the following information from each eligible study: the first author's name and related information, such as age, gender, lesion distribution (bilateral/unilateral, posterior/other, etc.), and chest CT findings (e.g., GGO, consolidation, peribronchial thickening). Moreover, the chest CT findings were classified as typical (GGO with or without superimposed consolidations in the posterior and periphery of both lungs), atypical (nodules, cavities, bronchiectasis, pleural or pericardial effusion, lymphadenopathies, halo sign, and reversed halo sign), and borderline (a combination of typical and atypical findings) [9, 10]. All findings were assessed and categorized according to a certified radiologist.

\section{Results}

\section{Literature search and screening}

The initial search yielded 1140 potential studies. After removing 329 duplicates, 811 articles were reviewed, by title and abstract, of which 742 articles were excluded. The full text of the remaining 69 articles was studied, and 34 articles were also excluded due to the lack of specific information on infants. Finally, the remaining 35 independent studies were used in the present study. A summary of the article selection process using the PRISMA flow chart is shown in Fig. 1.

\section{Data extraction}

Out of the 35 articles included in the study, 9 studies were cross-sectional [11-19], 11 case reports [20-30], and 15 case series [17, 19, 22, 31-42]. Among them, 28 studies were conducted in China, 4 studies in Iran, and 3 from Turkey, France, and Brazil. The largest study was a report by Sun et al. [16] in Wuhan, China, which included 36 infants with confirmed COVID-19 infection, but only 8 patients had chest CT scan results, which were included in our study. A total of nine variables were extracted from eligible studies (Supplemental Table 1).

Overall, 70 COVID-19-confirmed infants were studied. The mean age of the infants was 4.1 months, with a range of 


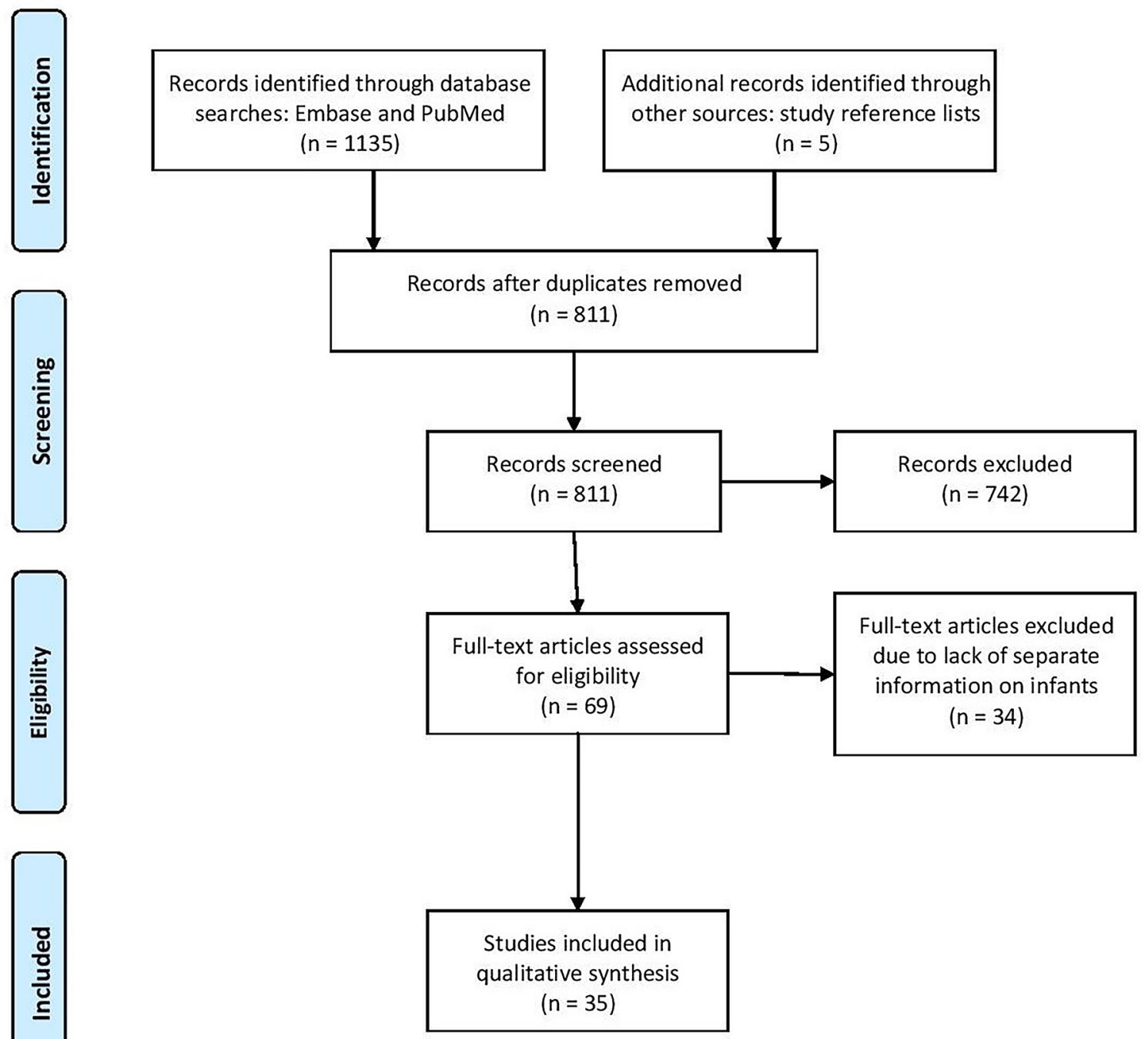

Fig. 1 Flow chart of the search and study inclusion

1 day to 12 months. Further, $58.5 \%$ of the individuals were male, with a male to female ratio of 1.4:1.

\section{Chest CT findings}

Out of the 70 patients studied, $88.57 \%$ (62/70) had abnormalities in the chest CT findings, and $11.43 \%(8 / 70)$ had a normal chest CT scan. The lesions' distribution on chest CT scan was reported in 59 patients where 25 patients had unilateral lung involvement, and 34 had bilateral lesions. The distribution of the lesions in three infants was not reported [40]. The chest CT images of 46 out of 70 patients were printed and re-examined and re-reported by an expert pediatric radiologist. For the remaining 24 patients, the original report of the chest CT scans which was published in the paper is presented.

The most common manifestation in chest CT scans was ground-glass opacity (GGO) in $50(71.43 \%)$ patients, followed by peribronchial thickening (42[60\%]), linear or band-shaped opacities and subsegmental collapse (21 [32.8\%]), consolidation (20 [28.57\%]), and nodule (13 [18.57\%]). Radiologic evidence of effusion and focal lucency was also reported in 5 (7.14\%) and 5 (7.14\%) patients, respectively (Fig. 2). Regarding lung involvement, most patients had typical involvement (32/59), followed by borderline (17/59) and atypical (10/59) involvement. The patterns of the lesions and main chest CT findings are described in detail in Supplemental Table 1. 

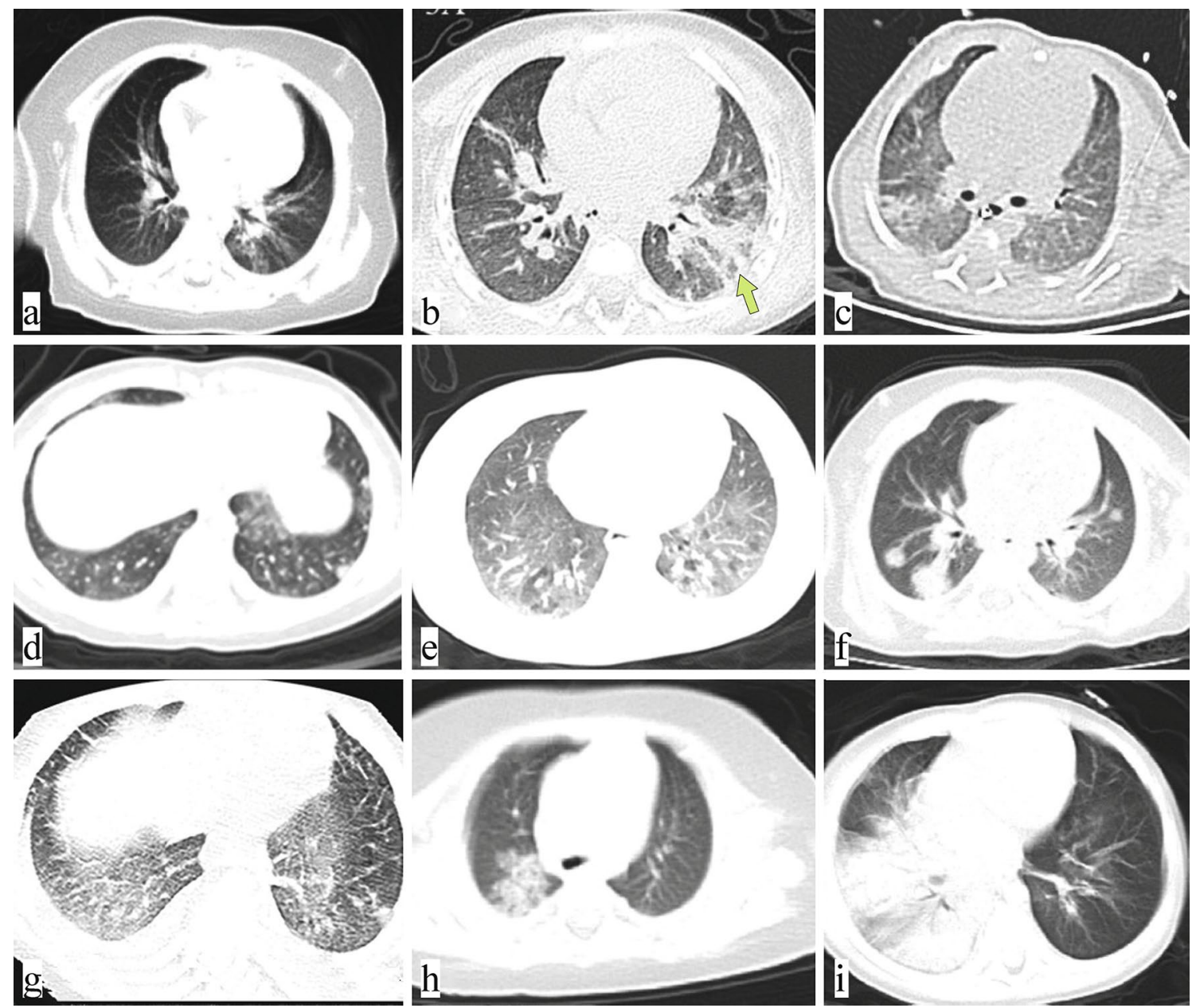

Fig. 2 High-resolution CT (HRCT) patterns of COVID-19 in infants: a-c lobar grand glass opacities with strip-like appearance, $\mathbf{d}-\mathbf{f}$ multinodular pattern with or without grand glass opacities or consolida-

\section{Discussion}

The global number of COVID-19 cases is increasing daily, and more children are getting infected. Several guidelines consider a chest CT scan as an essential modality in the diagnosis and follow-up of COVID-19 patients [43-45]. Therefore, it is important to investigate the typical and atypical findings of chest $\mathrm{CT}$ scan in different age groups.

Children with COVID-19 infection tend to have milder clinical symptoms and better prognosis in comparison to adults [46-48]. In addition, the imaging findings in the pediatric population show less severe abnormalities [7, 49], which can be attributed to several factors. The angiotensinconverting-enzyme (ACE) 2 receptor, which mediates the entry of the COVID-19 virus to the respiratory epithelial cells by interacting with the spike protein of the virus, plays an important role in the pathogenesis of the disease. The number of these receptors is relatively lower in younger populations compared with adults. Moreover, the ACE2 tion, $\mathbf{g}$ diffuse grand glass with septa thickening and unilateral rightsided pleural effusion, $\mathbf{h}$, i round pneumonia and lobar consolidation

receptors are mainly expressed in the lung tissues which may lead to milder symptoms and lesser multi-organ involvement, thus fewer critical cases among children. Besides, the ACE2 receptor is less mature and functional in children, making them less sensitive to the virus [50]. In addition, the developing immune system in children results in a weaker response to the virus in comparison to adults, leading to milder symptoms and to less severe lung injury $[4,51]$.

Of note, it has been suggested that even within the pediatric population, there are some differences among age groups. Although numerous studies have investigated the chest CT scan findings in children infected with COVID-19, in its entirety, no study has yet focused on specific pediatric populations. Infants, children younger than 12 months, are a vulnerable group in whom the clinical features and imaging findings of the COVID-19 infection are not fully understood. $\mathrm{Li}$ et al. showed that in patients under 18 years old, with age increase, the disease symptoms get more severe, and the abnormal findings in chest CT scan are more prominent, 
indicating greater lung involvement [52]. However, a largescale study on 2143 pediatric patients showed that the proportion of severe critical cases in infants was higher compared with their older counterparts, putting them at a higher risk of developing respiratory failure due to COVID19 infection [53]. However, in an analysis of the pediatric COVID-19 cases in the United States, the rate of hospitalization among the patients younger than 18 years other than infants was higher than that of infants [54]. Studies seem to show controversial results regarding lung involvement in different pediatric age groups and regarding the severity of clinical and imaging findings in neonates compared to other pediatric age groups. Therefore, special consideration should be given to the determination of clinical features, imaging findings, treatment strategies, and preventive measures in this population. Accordingly, for the first time, we conducted a systematic review of the literature to provide a better understanding of abnormal chest CT scan findings in infants infected with COVID-19.

In our study, GGO was found to be the most prevalent manifestation in chest CT scans of confirmed COVID-19 infants, followed by peribronchial thickening, consolidation, linear or band-shaped opacities, and lung nodules. The majority of cases had bilateral abnormalities, more commonly in the subpleural regions of the lung periphery. In the cases with unilateral lung involvement, the lesions were mainly located in the upper lobe of the right lung. Our results are in line with previous studies in the pediatric population which found GGO and consolidation as the most prevalent CT scan findings in children [55-59]. In a systematic review of studies on chest CT scan findings in children with COVID-19 infection, GGO and consolidation were reported in $32.9 \%$ and $6.5 \%$ of the patients, respectively [60]. In our systematic review, which is specifically limited to infants, GGO and consolidation were more frequent than the figures reported for the pediatric population in its entirety. Moreover, specific findings, such as peribronchial thickening, multiple lung nodules, and linear or band-shaped opacities, were more commonly reported in our included studies compared to adults and other pediatric age groups.

The presence of linear or band-shaped opacities in lung CT scans can be explained by increased septa and peribronchial thickening and the subsegmental collapse of the involved lung. As can be seen in other viral infections, such as that by the respiratory syncytial virus (RSV), the bronchial tree involvement presented as peribronchial thickening is more commonly observed in infants with COVID-19 infection than in adults. However, these observed differences should be interpreted with caution because we were not able to formally compare the frequency of different CT scan findings between infants and older children, which highlights the need for performing an original study specifically for this aim. In addition, about $11.5 \%$ of the PCR-positive patients included in our systematic review had a normal chest CT scan. They were mainly asymptomatic or had minimal upper respiratory symptoms and were suspected to have COVID19 because of the positive history of contact with other members of the family who were known cases of COVID19. It is worth noting that this is an underestimate of the frequency of normal chest CT scans; Patients with abnormal CT scan findings were more likely be reported. The exact pathophysiology for the explanation of these variations thus needs to be clarified in future studies.

In a study by Merkus et al., lung CT scan showed no abnormalities in 35\% of pediatric patients with COVID-19 infection who had no or minor symptoms, suggesting that lung CT scan is a weak screening tool in this population with a high rate of false negatives [61]. Chest CT scan also can be normal in the early stages of the disease, and a mismatch can be observed between the severity of symptoms and lung involvement [62]. Similarly, we saw that $11.43 \%$ of the included infants with COVID-19 symptoms had normal CT scans, although one of the cases developed abnormalities later in a follow-up image, suggesting a delay between respiratory symptoms and observable abnormal findings in CT scan. The same delay was reported in another study in which $5.3 \%$ of patients with confirmed COVID-19 infection who had a normal chest CT scan at baseline showed abnormal pulmonary findings $2-7$ days after the onset of the symptoms [63].

It should be noted that one study reported an infant with pre-existing bronchopulmonary dysplasia (BPD) and a history of prematurity who was diagnosed with the multisystem inflammatory syndrome in children (MIS-C), and the lung involvement seen in the CT scan was critical [23]. In another infant with a past surgical history for nephroblastoma and congenital heart disease, the disease presentation was critical; co-infection of COVID-19 and mycoplasma was detected, and the lung CT scan findings showed multiple pneumonia-like changes including blurred lung markings and multiple patchy consolidations in both lungs [17]. The presence of comorbidities is associated with poorer prognosis and increased mortality among both children and adults, so high-risk groups should be under more strict protections [46].

Our study has certain limitations. First, different CT scan protocols had been used in the included studies, which may be a potential confounder in the observed abnormalities. Second, our review was limited mostly to case reports and case series. Furthermore, no studies analyzed the difference between the chest $\mathrm{CT}$ findings in infants and in other age categories. Some studies indeed included infants with COVID-19 infection, but they did not report the individual data for the patients and provided only group-level statistics. In addition, most of the included studies were from China, limiting the generalization 
of our findings to other populations and highlighting the need for more evidence from other countries.

This study findings help to provide a better understanding of the variations of clinical features of COVID-19 disease and its associated chest CT findings in infants who are a vulnerable age group. Our results may thus assist pediatricians and radiologists with a more accurate diagnosis in this age group based on the chest CT scan.

In conclusion, this systematic review provides an analysis of the current literature on chest CT scan findings in COVID-19 pediatric patients younger than 1 year old. GGO and peribronchial thickening are the most prevalent findings in the chest CT scans of the included infants. Linear or band-shaped opacities, consolidation, as well as pulmonary nodules, are more common in infants in comparison to other populations. The high prevalence of peribronchial thickening and linear or band-shaped opacities in infantile COVID-19 infections suggests that the disease in this age group has the nature of atypical pneumonia, like other viral infections. In total, infantile COVID-19 infections can present with four manifestations of usual COVID-19 infections (peripheral GGO), atypical pneumonia (peribronchial thickening and linear or band-shaped opacities), lobar pneumonia (consolidation), and opportunistic infections, such as fungi (pulmonary nodules). Further studies are needed to evaluate chest CT findings in infants in more detail, and appropriate guidelines should be developed for the interpretation of the COVID-related chest CT scan changes concerning these observed differences.

Supplementary Information The online version of this article (https:// doi.org/10.1007/s12519-021-00424-1) contains supplementary material, which is available to authorized users.

Author contributions AG, SG, and MB contributed to study design. AG, AS, SA, MB, and EM contributed to the systematic search, screening, and data abstraction. SG, MB, MRB, AG, and EM contributed to writing the first draft. All the authors revised and approved the manuscript.

Funding The authors received no specific funding for this work.

\section{Compliance with ethical standards}

Ethical approval This article does not contain any studies with human participants or animals performed by any of the authors. All the data involved in this study were extracted from published articles.

Conflict of interest All the authors declared that they have no conflict of interest.

\section{References}

1. Yang X, Yu Y, Xu J, Shu H, Liu H, Wu Y, et al. Clinical course and outcomes of critically ill patients with SARS-CoV-2 pneumonia in Wuhan, China: a single-centered, retrospective, observational study. Lancet Respir Med. 2020;8:475-81.

2. Lu Q, Shi Y. Coronavirus disease (COVID-19) and neonate: What neonatologist need to know. J Med Virol. 2020;92:564-7.

3. Ai T, Yang Z, Hou H, Zhan C, Chen C, Lv W, et al. Correlation of chest CT and RT-PCR testing for coronavirus disease 2019 (COVID-19) in China: a report of 1014 cases. Radiology. 2020;296:E32-40.

4. Fang Y, Zhang H, Xie J, Lin M, Ying L, Pang P, et al. Sensitivity of chest CT for COVID-19: comparison to RT-PCR. Radiology. 2020;296:E115-7.

5. Zhao W, Zhong Z, Xie X, Yu Q, Liu J. Relation between chest $\mathrm{CT}$ findings and clinical conditions of coronavirus disease (COVID-19) pneumonia: a multicenter study. Am J Roentgenol. 2020;214:1072-7.

6. Brogna B, Bignardi E, Brogna C, Alberigo M, Grappone M, Megliola A, et al. Typical CT findings of COVID-19 pneumonia in patients presenting with repetitive negative RT-PCR. Radiography (Lond). 2020:S1078-8174:30199-1.

7. Azadbakht J, Haghi-Aminjan H, Farhood B. Chest CT findings of COVID-19-infected patients, are there differences between pediatric and adult patients? A systematic review. Egypt J Radiol Nucl Med. 2020;51:1-10.

8. Moher D, Liberati A, Tetzlaff J, Altman DG, Group P. Preferred reporting items for systematic reviews and meta-analyses: the PRISMA statement. PLoS med. 2009;6:e1000097.

9. Caruso D, Polidori T, Guido G, Nicolai M, Bracci B, Cremona A, et al. Typical and atypical COVID-19 computed tomography findings. World J Clin Cases. 2020;8:3177-87.

10. Salehi S, Abedi A, Radmard AR, Sorouri M, Gholamrezanezhad A. Chest computed tomography manifestation of coronavirus disease 2019 (COVID-19) in patients with cardiothoracic conditions. J Thorac Imaging. 2020;35:W90-6.

11. Du H, Dong X, Zhang JJ, Cao YY, Akdis M, Huang PQ, et al. Clinical characteristics of 182 pediatric COVID-19 patients with different severities and allergic status. Allergy. 2021;76:510-32.

12. Li Y, Wang H, Wang F, Du H, Liu X, Chen P, et al. Comparison of hospitalized patients with pneumonia caused by COVID19 and influenza A in children under 5 years. Int J Infect Dis. 2020;98:80-3.

13. Liu H, Liu F, Li J, Zhang T, Wang D, Lan W. Clinical and CT imaging features of the COVID-19 pneumonia: focus on pregnant women and children. J Infect. 2020;80:e7-13.

14. Ma H, Hu J, Tian J, Zhou X, Li H, Laws MT, et al. A singlecenter, retrospective study of COVID-19 features in children: a descriptive investigation. BMC Med. 2020;18:1-11.

15. Palabiyik F, Kokurcan SO, Hatipoglu N, Cebeci SO, Inci E. Imaging of COVID-19 pneumonia in children. Br J Radiol. 2020;93:20200647.

16. Sun D, Chen X, Li H, Lu XX, Xiao H, Zhang FR, et al. SARSCoV-2 infection in infants under 1 year of age in Wuhan City. China World J Pediatr. 2020;16:260-6.

17. Wu YT, Liu J, Xu JJ, Chen YF, Yang W, Chen Y, et al. Neonatal outcome in 29 pregnant women with COVID-19: A retrospective study in Wuhan. China PLoS med. 2020;17:e1003195.

18. Xia W, Shao J, Guo Y, Peng X, Li Z, Hu D. Clinical and CT features in pediatric patients with COVID-19 infection: Different points from adults. Pediatr Pulmonol. 2020;55:1169-74.

19. Zheng F, Liao C, Fan QH, Chen HB, Zhao XG, Xie ZG, et al. Clinical characteristics of children with coronavirus disease 2019 in Hubei, China. Curr Med Sci. 2019;2020:275-80.

20. Abasse S, Essabar L, Costin T, Mahistra V, Kaci M, Braconnier A, et al. Neonatal COVID-19 Pneumonia: report of the first case in a preterm neonate in Mayotte, an overseas department of France. Children. 2020;7:87-91. 
21. Cui Y, Tian M, Huang D, Wang X, Huang Y, Fan L, et al. A 55-Day-Old Female Infant infected with COVID 19: presenting with pneumonia, liver injury, and heart damage. J Infect Dis. 2020;221:1775-81.

22. Fan Q, Pan Y, Wu Q, Liu S, Song X, Xie Z, et al. Anal swab findings in an infant with COVID-19. Pediatr investig. 2020;4:48-50.

23. Farias EC, Justino MC, Mello ML. Multisystem inflammatory syndrome in a child associated with coronavirus disease 19 in the brazilian amazon: fatal outcome in an infant. Rev Paul Pediatr. 2020;38:e2020165.

24. Jafari R, Cegolon L, Torkaman M, Kashaki M, Dehghanpoor F, Cheraghalipoor F, et al. A 6 months old infant with fever, dyspnea and poor feeding, diagnosed with COVID-19. Travel Med Infect Dis. 2020;36:101789.

25. Li C, Luo F, Wu B. A 3-month-old child with COVID-19: a case report. Medicine. 2020;99:e20661.

26. Li J, Feng J, Liu TH, Xu FC, Song GQ. An infant with a mild SARS-CoV-2 infection detected only by anal swabs: a case report. Braz J Infect Dis. 2020;24:247-9.

27. Mahdavi S, Kheirieh A, Daliri S, Kalantar MH, Valikhani M, Khosravi A, et al. More reliability of suspicious symptoms plus chest CT-scan than RT_PCR test for the diagnosis of COVID-19 in an 18-days-old neonate. IDCases. 2020;21:e00905.

28. Navaeifar MR, Ghazaghi MP, Shahbaznejad L, Rouhanizadeh $\mathrm{H}$, Abutalebi M, Varandi MR, et al. Fever with rash is one of the first presentations of COVID-19 in children: a case report. Int Med Case Rep J. 2020;13:335.

29. Ekrami Noghabi M, Baniasad A, Heidari E, Davoudian N, Malekzadeh F. A 35-day old infant with COVID-19. Iran J Pediatr. 2020;30:e103807.

30. Wei Y, Liu X, Yuan J, Shi J, Zhang X, Wang D, et al. Coronavirus disease-2019 (COVID-19) infection in a 3-month-old infant: Clinical features, treatment and probable route of transmission. IDCases. 2020;22:e00937.

31. Cai X, Ma Y, Li S, Chen Y, Rong Z, Li W. Clinical Characteristics of 5 COVID-19 cases with non-respiratory symptoms as the first manifestation in children. Front Pediatr. 2020;8:258.

32. Huang L, Jiang J, Li X, Zhou Y, Xu M, Zhou J. Initial CT imaging characters of an imported family cluster of COVID-19. Clin Imaging. 2020;65:78-81.

33. Li A, Zhou X, Lu W, Zhou Y, Liu Q. COVID-19 in two infants in China. Immun Inflamm Dis. 2020;8:380-3.

34. Li W, Cui H, Li K, Fang Y, Li S. Chest computed tomography in children with COVID-19 respiratory infection. Pediatr radiol. 2020;11:1-4.

35. Liu M, Song Z, Xiao K. High-resolution computed tomography manifestations of 5 pediatric patients with 2019 novel coronavirus. J Comput Assist Tomogr. 2020;44:311-3.

36. Lu Y, Wen H, Rong D, Zhou Z, Liu H. Clinical characteristics and radiological features of children infected with the 2019 novel coronavirus. Clin Radiol. 2020;75:520-5.

37. Shen Q, Guo W, Guo T, Li J, He W, Ni S, Ouyang X, Liu J, Xie Y, Tan X, Zhou Z, Peng H. Novel coronavirus infection in children outside of Wuhan. China. Pediatr Pulmonol. 2020;55:1424-9.

38. Su L, Ma X, Yu H, Zhang Z, Bian P, Han Y, et al. The different clinical characteristics of corona virus disease cases between children and their families in China-the character of children with COVID-19. Emerg microbes Infect. 2020;9:707-13.

39. Feng X, Tao X, Zeng L, Wang W, Li G. Application of pulmonary ultrasound in the diagnosis of COVID-19 pneumonia in neonates. Zhonghua Er Ke Za Zhi. 2020;58:347-50.

40. Zhang ZJ, Yu XJ, Fu T, Liu Y, Jiang Y, Yang BX, et al. Novel coronavirus infection in newborn babies under 28 days in China. Eur Respir J. 2020;55:2000697.
41. Zhong Z, Xie X, Huang W, Zhao W, Yu Q, Liu J. Chest CT findings and clinical features of coronavirus disease 2019 in children. Zhong Nan Da Xue Xue Bao Yi Xue Ban. 2020;45:236-42.

42. Zhou Y, Yang GD, Feng K, Huang H, Yun YX, Mou XY, et al. Clinical features and chest CT findings of coronavirus disease 2019 in infants and young children. Zhongguo Dang Dai Er Ke Za Zhi. 2020;22:215-20.

43. Chen ZM, Fu JF, Shu Q, Chen YH, Hua CZ, Li FB, et al. Diagnosis and treatment recommendations for pediatric respiratory infection caused by the 2019 novel coronavirus. World J Pediatr. 2020;16:240-6.

44. Jin YH, Zhan QY, Peng ZY, Ren XQ, Yin XT, Cai L, et al. Chemoprophylaxis, diagnosis, treatments, and discharge management of COVID-19: an evidence-based clinical practice guideline (updated version). Mil Med Res. 2020;7:1-33.

45. Bischof E, Chen G, Ferretti MT. Understanding COVID-19 new diagnostic guidelines-a message of reassurance from an internal medicine doctor in Shanghai. Swiss Med Wkly. 2020;150:w20216.

46. Ludvigsson JF. Systematic review of COVID-19 in children shows milder cases and a better prognosis than adults. Acta Paediatr. 2020;109:1088-95.

47. Mantovani A, Rinaldi E, Zusi C, Beatrice G, Saccomani MD, Dalbeni A. Coronavirus disease 2019 (COVID-19) in children and/or adolescents: a meta-analysis. Pediatr Res. 2020. https:// doi.org/10.1038/s41390-020-1015-2.

48. Ghodsi A, Azarfar A, Ghahremani S. A review of coronavirus disease (COVID-19) in children. J Pediatr Nephrol. 2020;8:1-6.

49. Kumar J, Meena J, Yadav A, Yadav J. Radiological findings of COVID-19 in children: a systematic review and meta-analysis. J Trop Pediatr. 2020;1:1-8.

50. Yang ZD, Zhou GJ, Jin RM, Liu ZS, Dong ZQ, Xie X, et al. Clinical and transmission dynamics characteristics of 406 children with coronavirus disease 2019 in China: a review. J Infect. 2020;81:e11-5.

51. Du W, Yu J, Wang H, Zhang X, Zhang S, Li Q, et al. Clinical characteristics of COVID-19 in children compared with adults in Shandong Province. China Infection. 2020;48:445-52.

52. Liu W, Zhang Q, Chen J, Xiang R, Song H, Shu S, et al. Detection of Covid-19 in children in early January 2020 in Wuhan. China $\mathrm{N}$ Eng J Med. 2020;382:1370-1.

53. Dong Y, Mo X, Hu Y, Qi X, Jiang F, Jiang Z, et al. Epidemiological characteristics of 2143 pediatric patients with 2019 coronavirus disease in China. J Emerg Med. 2020;58:712-3.

54. CDC COVID-19 Response Team. Coronavirus Disease. in Children-United States, February 12-April 2, 2020. MMWR Morb Mortal Wkly Rep. 2019;2020:422-6.

55. Sun Z, Zhang N, Li Y, Xu X. A systematic review of chest imaging findings in COVID-19. Quant Imaging Med Surg. 2020;10:1058-79.

56. Ding Y, Yan H, Guo W. Clinical characteristics of children With COVID-19: a meta-analysis. Front Pediatr. 2020;8:431.

57. Dosanjh A. Pediatric coronavirus disease 2019 and radiographic screening: identification of patients at risk. Pediatr Allergy Immunol Pulmonol. 2020;33:53-6.

58. Di Nardo M, van Leeuwen G, Loreti A, Barbieri MA, Guner Y, Locatelli F, et al. A literature review of 2019 novel coronavirus (SARS-CoV2) infection in neonates and children. Pediatr Res. 2020. https://doi.org/10.1038/s41390-020-1065-5.

59. Duan YN, Zhu YQ, Tang LL, Qin J. CT features of novel coronavirus pneumonia (COVID-19) in children. Eur Radiol. 2020;30:4427-33.

60. Hoang A, Chorath K, Moreira A, Evans M, Burmeister-Morton F, Burmeister F, Naqvi R, Petershack M, Moreira A. COVID-19 in 7780 pediatric patients: A systematic review. EClinicalMedicine. 2020;24:100433. 
61. Merkus P, Klein WM. The value of chest CT as a COVID-19 screening tool in children. Eur Respir J. 2020; 55.2001241.

62. Zeng Y, Fu J, Yu X, Huang Z, Yin X, Geng D, et al. Should computed tomography (CT) be used as a screening or follow-up tool for asymptomatic patients with SARS-CoV-2 infection? Quant Imaging Med Surg. 2020;10:1150.
63. Cui N, Zou X, Xu L. Preliminary CT findings of coronavirus disease 2019 (COVID-19). Clin Imaging. 2020;65:124-32.

Publisher's Note Springer Nature remains neutral with regard to jurisdictional claims in published maps and institutional affiliations. 